Research Article

\title{
Some Studies in Hemirings by the Falling Fuzzy $k$-Ideals
}

\author{
R. Anjum $\mathbb{D},{ }^{1}$ F. Sarfraz $\mathbb{D},{ }^{1}$ N. Kausar $\mathbb{D},{ }^{2}$ Y. U. Gaba $\mathbb{D},{ }^{3,4,5}$ H. Aydi $\mathbb{D},{ }^{6,7,8}$ M. Munir $\mathbb{D}$, ${ }^{9}$ \\ and Salahuddin $\mathbb{D D}^{10}$ \\ ${ }^{1}$ Department of Mathematics \& Statistics, The University of Lahore, Lahore, Pakistan \\ ${ }^{2}$ Yildiz Technical University, Faculty of Arts and Science, Department of Mathematics, Esenler, 34210 Istanbul, Turkey \\ ${ }^{3}$ Quantum Leap Africa (QLA), AIMS Rwanda Centre, Remera Sector KN 3, Kigali, Rwanda \\ ${ }^{4}$ African Center for Advanced Studies, P.O. Box 4477, Yaounde, Cameroon \\ ${ }^{5}$ Institut de Mathematiques et de Sciences Physiques (IMSP/UAC), Laboratoire de Topologie Fondamentale, Computationnelle et \\ leurs Applications (Lab-ToFoCApp), BP 613, Porto-Novo, Benin \\ ${ }^{6}$ Université de Sousse, Institut Supérieur d'Informatique et des Techniques de Communication, H. Sousse 4000, Tunisia \\ ${ }^{7}$ Department of Mathematics and Applied Mathematics, Sefako Makgatho Health Sciences University, Ga-Rankuwa, South Africa \\ ${ }^{8}$ China Medical University Hospital, China Medical University, Taichung 40402, Taiwan \\ ${ }^{9}$ Department of Mathematics, Government Postgraduate College, Abbottabad, Pakistan \\ ${ }^{10}$ Department of Mathematics, Jazan University, Jazan, Saudi Arabia
}

Correspondence should be addressed to Y. U. Gaba; yaeulrich.gaba@gmail.com and H. Aydi; hassen.aydi@isima.rnu.tn

Received 16 May 2021; Revised 22 June 2021; Accepted 2 July 2021; Published 4 August 2021

Academic Editor: Shrideh K. Q. Al-Omari

Copyright $\odot 2021$ R. Anjum et al. This is an open access article distributed under the Creative Commons Attribution License, which permits unrestricted use, distribution, and reproduction in any medium, provided the original work is properly cited.

In this article, we establish the idea of falling fuzzy $k$-ideals in hemirings through the falling shadow theory and fuzzy sets. We shall express the relations between fuzzy $k$-ideals and falling fuzzy $k$-ideals in hemirings. In particular, we shall establish different characterizations of $k$ -hemiregular hemirings in the perfect positive correlation and independent probability space by means of falling fuzzy $k$-ideals.

\section{Introduction}

Semiring theory had been developed by Vandiver in order to generalize rings [1]. Semirings are used in major applied mathematical fields, like automata and computer sciences $[2,3]$. In the structural theory of hemirings and other algebraic structures, like BCI-algebras and KU-algebras, ideals play a significant role in their studies. Several types of ideals like $p$-ideals, $a$-ideals, and $q$-ideals in BCI-algebras and KUalgebras have been discussed by Senapati and others in $[4$, 5]. The type of ideals, viz., $m$-ideals, was studied by Munir and others in [6] for semigroups and in [7] for semirings. In [8], Henriksen defined finite category of $k$-ideals related to the ideals in semirings. In [9], La Torre examined the $h$ -ideals and $k$-ideals in hemirings.

Several authors have studied fuzzy $h$-ideals and fuzzy $k$ -ideals in semirings [10, 11]. In [12], Shabir and Anjum have examined the fuzzy right $k$-ideals by using the characterizations of right $k$-ideals in hemirings. Prime $h$-ideals and semiprime $k$ -bi-ideals are also defined in hemirings in $[13,14]$. The fuzzy sets can be used to study patterns in classification and information processing. The idea of the fuzzy sets has broader applicability, e.g., Goodman worked on the equivalence between a class of random sets and a fuzzy set [15]. In [16-19], Verma used the fuzzy sets in the multiple attribute decision-making and multicriteria decision making environments to study different problems. Some more applications of the fuzzy sets are found in the context of controlled fuzzy metric spaces [20] (see also [21-23]) and pattern recognition [24].

The relationship between probability theory and fuzzy sets is one of most contentious matters in an information science and an uncertainty modeling. The literature on this debate is obscure. Dubois and Prade, in their article [25], have addressed some traditional incomprehension between 
fuzzy sets and probabilities. They considered probabilistic analysis of membership functions that helped in assessments of these functions. In 1981, a statistical experiment was conducted to determine the membership function of the fuzzy concept "young" by three different groups of students under the supervision of Wang in China. From that experiment, they approached the existence of the resulting membership function in the theory of fuzzy sets. After this, Wang developed this approach further and furnished a precise mathematical structure of this theory in his book [26]. In [27], Tan and others established a conceptual perspective to define a fuzzy inference relation based on the theory of falling shadows. In [28, 29], Jun and Yu established some connections between the probability theory and the fuzzy mathematics in algebraic structures, like BCK-algebras and hemirings.

In a future work, one can consider $k$-hemiregular using falling Yuan and Lee, [30], considered a fuzzy subgroup (subring, ideal) as the falling shadow of a cloud of the subgroups (subrings, ideals). By taking inspiration from [30], the notion of falling fuzzy $k$-ideals of a hemiring is introduced on the basis of the theory of falling shadows and fuzzy sets. This theory is widely applicable in information science and intelligent and fuzzy systems.

This research article is arranged such that the first section briefly describes the history and reviews the literature of fuzzy set theory, ideals in hemirings, and the theory of falling shadows. The second section provides an explanation of the fundamental concepts such as hemirings, ideals, fuzzy sets, and probability spaces. The falling fuzzy $k$-ideals in a probability space and their results are presented in Section III with intriguing examples. Section IV establishes characterizations of the $k$-hemiregular hemirings by a perfect positive correlation and an independent probability space through falling fuzzy $k$-ideals. Finally, the research is concluded in the fifth section.

\section{Preliminaries}

Definition 1. A nonempty set $T$, together with two binary operations $(+)$ and $(\cdot)$, is called a semiring if $T$ is a semigroup under both the operations satisfying the following two distributive laws [13]:

$$
t^{1} \cdot\left(t^{2}+t^{3}\right)=t^{1} \cdot t^{2}+t^{1} \cdot t^{3} \text { and }\left(t^{1}+t^{2}\right) \cdot t^{3}=t^{1} \cdot t^{3}+t^{2} \cdot t^{3},
$$

for all $t^{1}, t^{2}, t^{3} \in T$.

Definition 2. A semiring $(T,+, \cdot)$ is a hemiring if $(T,+)$ is a commutative semigroup with a zero, i.e., $0 \in T$ such that $t^{1}$ $+0=0+t^{1}=t^{1}$ and $t^{1} \cdot 0=0 \cdot t^{1}=0$ for all $t^{1} \in T$ [13]

Definition 3. $e ́ \in T$ is the identity element of $T$ if $\dot{e} \cdot t^{1}=t^{1} \cdot \dot{e}=t^{1} \forall t^{1} \in T$ [12].

A hemiring $(T,+, \cdot)$ is commutative if $(T, \cdot)$ is a commutative semigroup [12].
Definition 4. Let $X \neq \phi$ be a subset of hemiring $T$. It is called an left (right) ideal of $T$ if

(i) $\dot{i}+j \in X$ for all $i, j \in X$

(ii) $r \dot{i} \in X(\dot{i} r \in X)$ for all $\dot{i} \in X, r \in T$

If $X$ is both a left and a right ideal of $T$, then it is called two-sided ideal or simply an ideal of $T$ [12].

Definition 5. Referring to $[12,13]$, the left (right) ideal $X$ in a hemiring $T$ is called a left (right) $k$-ideal of $T$ if $\dot{x}_{1}, \dot{y}_{1} \in X$ and

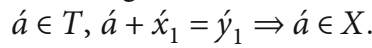

Definition 6. As in $[12,13]$, the $k$-closure $\bar{X}$ of $X$ in the hemiring $T$ is defined as

$$
\bar{X}=\left\{a \in T \mid a+x_{i}=x_{j} \text { for all } x_{i}, x_{j} \in X\right\} .
$$

Definition 7 . Let $F$ be a nonempty set. A membership function $\vartheta: F \longrightarrow[0,1]$ is called a fuzzy subset $\vartheta$ of $F$. Also, $\vartheta \neq$ $\phi$ if $\vartheta(\dot{x})>0$ for all $\dot{x}_{1} \in F[12]$.

Definition 8 [13]. A fuzzy set $\alpha$ in a hemiring $T$ is the fuzzy left (right) $k$-ideal of $T$ if for $e, \hat{f}, \dot{t}, \dot{t} \in T$, and we have the following:

(i) $\dot{\alpha}(\dot{e}+\dot{f}) \geq\{\dot{\alpha}(\dot{e}) \wedge \dot{\alpha}(\dot{f})\}$

(ii) $\dot{\alpha}(e ́ f) \geq \dot{\alpha}(\dot{f}),(\dot{\alpha}(e ́ f) \geq \dot{\alpha}(e ́))$

(iii) $\dot{e}+\dot{t}=\dot{t} \longrightarrow \dot{\alpha}(\dot{e}) \geq\{\dot{\alpha}(\hat{t}) \wedge \dot{\alpha}(\hat{t})\}$

Also, $\alpha$ is said to be a fuzzy $k$-ideal of $T$ if it is both a left $k$ -ideal and a right $k$-ideal of $T$.

Recall that $\dot{\alpha}(0) \geq \dot{\alpha}(\dot{e})$ for all $e \in T$.

Now, we spread some light on theory of the falling shadows. For more details, the references $[15,27]$ can be followed.

Let $V$ be a universal set and $R(v)$ be a power set of $V$. For $v \in V$, consider

$$
\dot{v}=\{F \mid v \in F, F \subseteq V\} .
$$

Let $F \in R(V)$, and then

$$
\dot{F}=\{\dot{v} \mid v \in F\} .
$$

If $A$ is a $\delta$-field in $R$ and $\subseteq A$, then the ordered pair $(R($ $V), A$ ) is called a hypermeasurable structure on $V$ [27].

Let $(\Lambda, \beta, R)$ be a probability space and $(R(V), A)$ be a hypermeasurable structure, then a random set on $V$ is considered as a function $\zeta: \Lambda \longrightarrow R(V)$ [27], which is $\beta-A$ measurable, i.e.,

$$
\zeta^{-1}(D)=\{\mathrm{q} \mid \mathrm{q} \in \Lambda, \zeta(\mathrm{q}) \in D\} \in ß, \forall D \in A .
$$

Definition 9. Referring to [27], let $\zeta$ be a random set on $V$, 
and then $\tilde{K}$ is called a falling shadow of $\zeta$ if

$$
\tilde{\mathrm{K}}(v)=R(\mathrm{q} \mid v \in \zeta(\mathrm{q})) \text {, for all } v \in V
$$

and $\zeta$ is cloud of $\tilde{\mathrm{K}}$, where $R$ is a probability measure.

Example 1. Given $(\Lambda, \beta, R)=\{[0,1], \beta, n\}$, where $\beta$ is a Borel field on $[0,1]$ and $n$ is the Lebesgue measure, suppose $\tilde{K}$ is a fuzzy set of $V$ and $\tilde{K}_{s}=\{v \in V \mid \tilde{K}(v) \geq s\}$ is a $s$-cut of $\tilde{K}$, then

$$
\zeta:[0,1] \longrightarrow R(V), s \mapsto \tilde{\mathrm{K}}_{s}
$$

is a random set, and $\zeta$ is a cloud of $\tilde{K}$. We shall call $\zeta$ as a cut cloud of $\tilde{K}$. It was defined in [29].

\section{Falling Fuzzy $k$-Ideals in a Probability Space}

In this section, we define the falling fuzzy $k$-ideals in a hemiring through a probability space. We shall also study the relation between fuzzy $k$-ideals and falling fuzzy $k$-ideals in a probability space.

Definition 10 [29]. Suppose $(\Lambda, \beta, R)$ is a probability space and $\zeta: \Lambda \longrightarrow R(T)$ is a random set, let $\zeta(q)$ be a left (right) ideal of $T$ for all $q \in \Lambda$, and then the random set of the falling shadow

$$
\tilde{\mathrm{K}}(x)=R(\mathrm{q} \mid x \in \zeta(\mathrm{q}))
$$

is known as a falling fuzzy left (right) ideal of $T$. Also, $\tilde{K}(x)$ is said to be a falling fuzzy ideal of $T$ if it is both a falling fuzzy left ideal and a falling fuzzy right ideal of $T$.

Let $(\Lambda, B, R)$ be a probability space. Given $\breve{G}(T)=\{g \mid g$ $: \Lambda \longrightarrow T\}$, where $T$ is a hemiring. Define the two operations $(+)$ and $(\cdot)$ on $\breve{G}(T)$ by

$$
\left(g_{1}^{b}+g_{2}^{b}\right)(\mathrm{q})=g_{1}^{b}(\mathrm{q})+g_{2}^{b}(\mathrm{q})
$$
$T)$.

$\left(g_{1}^{b} \cdot g_{2}^{b}\right)(\mathrm{q})=g_{1}^{b}(\mathrm{q}) \cdot g_{2}^{b}(\mathrm{q})$, where $q \in \Lambda$ and $g_{1}^{b}, g_{2}^{b} \in \breve{G}($

Let $\varphi \in \breve{G}(T)$ be defined by $\varphi(q)=0$ for each $q \in \Lambda$, and then $(\breve{G}(T),+, \cdot, \varphi)$ is a hemiring.

For any subset $B$ of $T$ and $g \in \breve{G}(T)$, consider

$$
B_{g}=\{\mathrm{q} \in \Lambda \mid g(\mathrm{q}) \in B\}
$$

Let

$$
\zeta: \Lambda \longrightarrow R(\breve{G}(T))
$$

be given as

$$
\mathrm{q} \mapsto\{g \in \breve{G}(T) \mid g(\mathrm{q}) \in B\}
$$

Then, $B_{g} \in \beta$.
Definition 11. Suppose $(\Lambda, \beta, R)$ is a probability space and $\zeta$ $: \Lambda \longrightarrow R(T)$ is a random set, if $\zeta(q)$ is a $k$-ideal of $T$ for all $q \in \Lambda$, then the falling shadow of the random set $\zeta$ given as

$$
\tilde{\mathrm{K}}(v)=R(\mathrm{q} \mid v \in \zeta(\mathrm{q}))
$$

is called the falling fuzzy $k$-ideal of $T$.

Proposition 1. We have that $\zeta(q)=\{g \in \breve{G}(T) \mid g(q) \in B\}$ is a $k$-ideal of $\breve{G}(T)$ for any $k$-ideal $B$ of $T$.

Proof. For $q \in \Lambda$, let $e, f \in \breve{G}(T)$ be such that $e, f \in \zeta(\mathrm{q})$, then $e(\mathrm{q}), f(\mathrm{q}) \in B$. As $B$ is a $k$-ideal of $T$, then $e(\mathrm{q})+f(\mathrm{q}) \in B$. Note that

$$
(e+f)(\mathrm{q})=e(\mathrm{q})+f(\mathrm{q}) \in B
$$

This implies that $e+f \in \zeta(\mathrm{q})$.

Let $e \in \zeta(\mathrm{q})$ and $s \in \breve{G}(T)$, then $e(\mathrm{q}) \in B$. Since $B$ is a $k$ -ideal of $T$, one has

$$
(s \cdot e)(\mathrm{q})=s(\mathrm{q}) \cdot e(\mathrm{q}) \in B
$$

Therefore, $s \cdot e \in \zeta(\mathrm{q})$, which shows that $\breve{G}(T) \cdot \zeta(\mathrm{q}) \in \zeta($ q). Similarly, we can also show that $\zeta(\mathrm{q}) \cdot \breve{G}(T) \in \zeta(\mathrm{q})$. Let , $f \in \zeta(\mathrm{q})$, and $s \in \breve{G}(T)$, then $f(\mathrm{q}) \in B$. Hence,

$$
(s+e)(\mathrm{q})=s(\mathrm{q})+e(\mathrm{q})=f(\mathrm{q})
$$

where $B$ is a $k$-ideal of $T$; so, $s(\mathrm{q}) \in B$ and also $s \in \zeta(\mathrm{q})$. Hence, $\zeta(\mathrm{q})$ is a $k$-ideal of $\breve{G}(T)$.

By the above result, $\tilde{K}$ is a falling fuzzy ideal of $\breve{G}(T)$ in $(\Lambda, B, R)$, where

$$
\tilde{\mathrm{K}}(f)=R(\mathrm{q} \mid f(\mathrm{q}) \in B\}
$$

Since

$$
\zeta^{-1}(f)=\{\mathrm{q} \in \Lambda \mid f \in \zeta(\mathrm{q})\}=\{\mathrm{q} \in \Lambda \mid f(\mathrm{q}) \in B\}=B_{f} \in B,
$$

one asserts that $\zeta$ is a random set on $\breve{G}(T)$; so, $\tilde{\mathrm{K}}$ is a falling fuzzy $k$-ideal on $T$ by Proposition 1.

We present the following examples.

Example 2. Consider the set $T=\left\{0, e^{b}, f^{b}, g^{b}\right\}$ equipped with the two operations $(+)$ and $(\cdot)$ defined by (see Table 1 ) 
Then, $(T,+, \cdot)$ is a hemiring [29]. Given $(\Lambda, B, R)=([0$ $, 1], B, n)$. Let $\zeta:[0,1] \longrightarrow R(T)$ be defined by

$$
\zeta(s)= \begin{cases}\{0\}, & \text { if } s \in[0,0.3), \\ \left\{0, e^{b}\right\}, & \text { if } s \in[0.3,0.5), \\ \left\{0, f^{b}\right\} & \text { if } s \in[0.5,0.7), \\ \left\{0, e^{b}, f^{b}\right\}, & \text { if } s \in[0.7,0.9), \\ T, & \text { if } s \in[0.9,1] .\end{cases}
$$

Then, $\zeta(s)$ is a $k$-ideal of $T$ for all $s \in[0,1]$. Hence, $\tilde{K}=$ $R(s \mid w \in \zeta(s))$ is a falling fuzzy $k$-ideal of $T$.

Example 3. Let $T=\left\{0, e^{b}, x^{b}, y^{b}, z^{b}\right\}$ be a hemiring with the two operations $(+)$ and $(\cdot)$ defined by the following Cayley table (see Table 2):

Then, $(T,+, \cdot)$ is a hemiring. Given $(\Lambda, \beta, R)=([0,1], \beta$ $, n)$. Define $\zeta:[0,1] \longrightarrow R(T)$ by

$$
\zeta(s)= \begin{cases}\{0\}, & \text { if } s \in[0,0.1), \\ \left\{0, x^{b}\right\} & \text { if } s \in[0.1,0.3), \\ \left\{0, z^{b}\right\}, & \text { if } s \in[0.3,0.5), \\ \left\{0, x^{b}, z^{b}\right\}, & \text { if } s \in[0.5,0.7), \\ \left\{0, e^{b}, x^{b}, y^{b}\right\}, & \text { if } s \in[0.7,0.9), \\ T, & \text { if } s \in[0.9,1] .\end{cases}
$$

Then, $\zeta(s)$ is a $k$-ideal of $T$ for all $s \in[0,1]$. Hence, $\tilde{K}=$ $R(s \mid w \in \zeta(s))$ is a falling fuzzy $k$-ideal of $T$.

Theorem 1. Each fuzzy $k$-ideal of $T$ is also a falling fuzzy $k$ -ideal of $T$, but the converse is not true.

Proof. Let $(\Lambda, B, R)=([0,1], B, n)$ be a probability space, i.e., $R$ is a probability measure, $B$ is a Bored field in $[0,1]$, and $n$ is the Lebesgue measure. Let $\mu$ be a fuzzy $k$-ideal of $T$, and then $\mu_{s}$ is a $k$-ideal of $T$ for all $s \in[0,1]$. Let $\zeta:[0,1] \longrightarrow R($ $T)$ be a random set given as $\zeta(s)=\mu_{s}$ for all $s \in[0,1]$. Hence, $\mu$ is also a falling fuzzy $k$-ideal of $T$.

For the converse, consider the hemiring $T=\left\{0, x^{b}, y^{b}\right.$, $\left.z^{b}\right\}$ with the two following binary operations $(+)$ and $(\cdot)$ (see Table 3): by

Given $(\Lambda, \beta, R)=([0,1], B, n)$. Define $\zeta:[0,1] \longrightarrow R(T)$

$$
\zeta(s)= \begin{cases}\{0\}, & \text { if } s \in[0,0.3), \\ \left\{0, x^{b}\right\}, & \text { if } s \in[0.3,0.5) \\ \left\{0, z^{b}\right\}, & \text { if } s \in[0.5,0.9), \\ T, & \text { if } s \in[0.9,1] .\end{cases}
$$

Then, $\zeta(s)$ is a $k$-ideal of $T$ for all $s \in[0,1]$. Hence, $\tilde{K}=$ $R(s \mid e \in \zeta(s))$ is the falling fuzzy $k$-ideal of $T$, which is represented by

$$
\begin{gathered}
\tilde{\mathrm{K}}(e)= \begin{cases}1, & \text { if } e=0, \\
0.3 & \text { if } e=x^{b}, \\
0.5 & \text { if } e=z^{b}, \\
0.1 & \text { if } e=y^{b},\end{cases} \\
\tilde{\mathrm{K}}_{s}= \begin{cases}\{0\}, & \text { if } s \in(0.5,1], \\
\left\{0, z^{b}\right\}, & \text { if } s \in(0.3,0.5], \\
\left\{0, x^{b}, z^{b}\right\}, & \text { if } s \in(0.1,0.3], \\
T, & \text { if } s \in(0,0.1] .\end{cases}
\end{gathered}
$$

If $s \in(0.1,0.3]$, then $\tilde{K}_{s}=\left\{0, x^{b}, z^{b}\right\}$ is not a $k$-ideal of $T$ since $x^{b}+z^{b}=y^{b} \mathrm{U}\left\{0, x^{b}, z^{b}\right\}$. Thus, $\tilde{K}$ is not a fuzzy $k$-ideal of $T$.

Consider the probability space $(\Lambda, B, R)$ and the falling shadow of a random set $\zeta: \Lambda \longrightarrow R(T)$. Let $\Lambda(e, \zeta)=\{\mathrm{q} \epsilon$ $\Lambda \mid w \in \zeta(\mathrm{q})\}$, then $\Lambda(e, \zeta) \in R$, where $e \in T$.

Theorem 2. If the falling shadow $\tilde{K}$ of the random set $\zeta: \Lambda$ $\longrightarrow R(T)$ is the falling fuzzy left (right) $k$-ideal in $T$ for all $e$ $, f, x, y \in T$, then we have the following assertions:

(i) $\Lambda(e, \zeta) \cap \Lambda(f, \zeta) \subseteq \Lambda(e+f, \zeta)$

(ii) $\Lambda(f, \zeta) \subseteq \Lambda(e f, \zeta),(\Lambda(e, \zeta) \subseteq \Lambda(e f, \zeta))$

(iii) $e+\dot{x}=\dot{y}$ implies that $\Lambda(\dot{x}, \zeta) \cap \Lambda(\dot{y}, \zeta) \subseteq \Lambda(e, \zeta)$

Proof.

(i) Let $q \in \Lambda(e, \zeta) \cap \Lambda(f, \zeta)$ where $e, f \in \zeta(\mathrm{q})$. Since $\zeta(\mathrm{q})$ is a left $k$-ideal in $T$, we have $e+f \in \zeta(\mathrm{q})$, so

$\mathrm{q} \in \Lambda(e+f, \zeta) \Rightarrow \Lambda(e, \zeta) \cap \Lambda(f, \zeta) \subseteq \Lambda(e+f, \zeta)$.

Similarly, the statement is true when $\zeta(\mathrm{q})$ is a right $k$ -ideal.

(ii) Suppose $q \in \Lambda(f, \zeta)$ and $f \in \zeta(\mathrm{q})$, then $\zeta(\mathrm{q})$ is a left $k$ -ideal on $T$, so $e f \in \zeta(\mathrm{q})$ and also $q \in \Lambda(e f, \zeta)$. It shows that 


$$
\Lambda(f, \zeta) \subseteq \Lambda(e f, \zeta)
$$

We can also prove

$$
\Lambda(e, \zeta) \subseteq \Lambda(e f, \zeta)
$$

is for right $k$-ideals.

(iii) Let $e+\dot{x}=\dot{y}$. Suppose $q \in \Lambda(\dot{x}, \zeta) \cap \Lambda(\dot{y}, \zeta)$ and $e \in$ $T$, then $\dot{x}, \dot{y} \in \zeta(\mathrm{q})$. As $\zeta(\mathrm{q})$ is a left $k$-ideal on $T$, so $e \in \zeta(\mathrm{q})$ and $\mathrm{q} \in \Lambda(e, \zeta)$. We have

$$
\Lambda(\dot{x}, \zeta) \cap \Lambda(\dot{y}, \zeta) \subseteq \Lambda(e, \zeta)
$$

Theorem 3. If $\tilde{K}$ is a falling fuzzy $k$-ideal of $T$, then $\tilde{K}(e f)$ $\geq \tilde{K}(e) \vee \tilde{K}(f)$.

Proof. Since $\Lambda(e f, \zeta) \supseteq \Lambda(e, \zeta) \cap \Lambda(f, \zeta)$, one writes

$$
\begin{aligned}
\tilde{\mathrm{K}}(e f) & =R(\mathrm{q} \mid e f \in \zeta(\mathrm{q})) \geq R(\mathrm{q} \mid e \in \zeta(\mathrm{q})) \cap(\mathrm{q} \mid f \in \zeta(\mathrm{q})) \\
& \geq R(\mathrm{q} \mid e \in \zeta(\mathrm{q}))+R(\mathrm{q} \mid f \in \zeta(\mathrm{q}))-R(\mathrm{q} \mid e \in \zeta(\mathrm{q})) \cap(\mathrm{q} \mid f \in \zeta(\mathrm{q})) \\
& \geq R(\mathrm{q} \mid e \in \zeta(\mathrm{q})) \vee R(\mathrm{q} \mid f \in \zeta(\mathrm{q}))=\tilde{\mathrm{K}}(e) \vee \tilde{\mathrm{K}}(f) .
\end{aligned}
$$

\section{Characterizations of $k$ - Hemiregular Hemirings}

In this section, we shall characterize the $k$-hemiregular hemirings on a perfect positive correlation probability space (PPS) and an independent probability space (IPS) through falling fuzzy $k$-ideals.

Definition 12 [13]. A hemiring $T$ is called a $k$-hemiregular if for all $x \in T$, there are $a_{1}, a_{2} \in T$ such that

$$
x+x a_{1} x=x a_{2} x .
$$

Lemma 1 [13]. If $X$ and $Y$ are both a right $k$-ideal and a left $k$ -ideal in a hemiring $T$, then

$$
\overline{X Y} \subseteq X \cap Y .
$$

Lemma 2. [13]. The hemiring $T$ is a hemiregular hemiring if for each right and left $k$-ideal $X$ and $Y$, we have $\overline{X Y}=X \cap Y$

Definition 13 . The probability space is called a perfect positive correlation [29] if

$$
\Lambda(e, \zeta) \subseteq \Lambda(f, \zeta) \text { or } \Lambda(f, \zeta) \subseteq \Lambda(e, \zeta) \forall e, f \in T .
$$

Definition 14. Suppose $\Lambda$ is a PPS, let $\tilde{K}_{\phi}$ and $\tilde{K}_{\psi}$ be the falling fuzzy $k$-ideals of $T$, and then the $I$ product of $\tilde{K}_{\phi}$ and $\tilde{K}_{\psi}$ is defined by

$$
\left(\tilde{\mathrm{K}}_{\phi} e_{I} \tilde{\mathrm{K}}_{\psi}\right)(e)={ }^{e+} \sum_{r=1}^{u} s_{r} t_{r}=\sum_{m=1}^{v} s^{\prime}{ }_{m} t^{\prime}{ }_{m}\left(\tilde{\mathrm{K}}_{\phi}\left(s_{r}\right) \wedge \tilde{\mathrm{K}}_{\phi}\left(\dot{s}_{m}\right) \wedge \tilde{\mathrm{K}}_{\psi}\left(t_{r}\right) \wedge \tilde{\mathrm{K}}_{\psi}\left(\hat{t}_{m}\right)\right)
$$

and $\quad\left(\tilde{\mathrm{K}}_{\phi} \mathrm{e}_{I} \tilde{\mathrm{K}}_{\psi}\right)(e)=0$ if $e$ cannot expressed as $e+\sum_{r=1}^{u} s_{r} t_{r}=$ $\sum_{m=1}^{v} \dot{s}_{m} \hat{t}_{m}$, where $r=1, \cdots, u$ and $, m=1, \cdots, v$.

Theorem 4. Suppose $\Lambda$ is a PPS and $\tilde{K}$ is the falling fuzzy left (right) $k$-ideal on $T$ for all $e, f, x, y \in T$, then

(1) $\tilde{K}(e+f) \geq \tilde{K}(e) \wedge \tilde{K}(f)$

(2) $\tilde{K}(e f) \geq \tilde{K}(f),(\tilde{K}(e f) \geq \tilde{K}(e))$

(3) $e+x=y \Rightarrow \tilde{K}(e) \geq \tilde{K}(x) \wedge \tilde{K}(y)$

Proof.

(1) Let $\zeta(\mathrm{q})$ be a $k$-ideal on $T$ for any $\mathrm{q} \in \Lambda$. Since

$$
\Lambda(\mathrm{e}, \zeta) \cap \Lambda(\mathrm{f}, \zeta) \subseteq \Lambda(\mathrm{e}+\mathrm{f}, \zeta),
$$

one writes

$$
\tilde{\mathrm{K}}(e+f)=R(\mathrm{q} \mid e+f \in \zeta(\mathrm{q})) \geq R(\{\mathrm{q} \mid e \in \zeta(\mathrm{q})\} \cap\{\mathrm{q} \mid f \in \zeta(\mathrm{q})\}) .
$$

If $\{\mathrm{q} \mid e \in \zeta(\mathrm{q})\} \supseteq\{\mathrm{q} \mid f \in \zeta(\mathrm{q})\}$, then $\tilde{\mathrm{K}}(e+f) \geq \tilde{\mathrm{K}}(e)$. While if $\{\mathbf{q} \mid e \in \zeta(\mathrm{q})\} \subseteq\{\mathrm{q} \mid f \in \zeta(\mathrm{q})\}$, then $\tilde{\mathrm{K}}(e+f) \geq \tilde{\mathrm{K}}(f)$. Hence,

$$
\tilde{\mathrm{K}}(e+f) \geq\{\tilde{\mathrm{K}}(e) \wedge \tilde{\mathrm{K}}(f)\} .
$$

(2) As $\zeta(\mathrm{q})$ is a left $k$-ideal on $T$, for any $\mathrm{q} \in \Lambda$, so $\Lambda(f$, $\zeta) \subseteq \Lambda(e f, \zeta)$. Hence, we have

$$
\tilde{\mathrm{K}}(e f)=R(\mathrm{q} \mid e f \in \zeta(\mathrm{q})) \geq R(\mathrm{q} \mid f \in \zeta(\mathrm{q}))=\tilde{\mathrm{K}}(f) .
$$

Similarly, for a right $k$-ideal, the statement is true.

(3) If $\zeta(\mathrm{q})$ is a left $k$-ideal on $T$ for any $\mathrm{q} \in \Lambda$, then

$$
e+x=y \text { implies that } \Lambda(x, \zeta) \cap \Lambda(y, \zeta) \subseteq \Lambda(e, \zeta) \text {. }
$$

So, we have

$$
\tilde{\mathrm{K}}(e)=R(\mathrm{q} \mid e \in \zeta(\mathrm{q})) \geq R(\{\mathrm{q} \mid x \in \zeta(\mathrm{q})\} \cap\{\mathrm{q} \mid y \in \zeta(\mathrm{q})\}) .
$$


If $\{\mathrm{q} \mid x \in \zeta(\mathrm{q})\} \supseteq\{\mathrm{q} \mid y \in \zeta(\mathrm{q})\}$, then $\tilde{\mathrm{K}}(e) \geq \tilde{\mathrm{K}}(x)$. While if $\{\mathrm{q} \mid x \in \zeta(\mathrm{q})\} \subseteq\{\mathrm{q} \mid y \in \zeta(\mathrm{q})\}$, then $\tilde{\mathrm{K}}(e) \geq \tilde{\mathrm{K}}(y)$. Hence,

$$
\tilde{\mathrm{K}}(e) \geq\{\tilde{\mathrm{K}}(x) \wedge \tilde{\mathrm{K}}(y)\} .
$$

Proposition 2 [27]. If $\Lambda$ is a perfect positive correlation probability space, then

$$
\left(\tilde{K}_{\phi} \cap \tilde{K}_{\psi}\right)(e)=\tilde{K}_{\phi}(e) \wedge \tilde{K}_{\psi}(e) .
$$

Proposition 3. Let $\Lambda$ be a (PPS) perfect positive correlation probability space. Suppose that $\tilde{K}_{\phi}$ and $\tilde{K}_{\psi}$ are falling fuzzy left (right) $k$-ideals on $T$, then $\tilde{K}_{\phi} \cap \tilde{K}_{\psi}$ is the falling fuzzy left (right) $k$-ideal on $T$.

Proof.

(1) We have

$$
\begin{aligned}
\left(\tilde{\mathrm{K}}_{\phi} \cap \tilde{\mathrm{K}}_{\psi}\right)(e+f) & =\tilde{\mathrm{K}}_{\phi}(e+f) \wedge \tilde{\mathrm{K}}_{\psi}(e+f) \\
& \geq\left\{\tilde{\mathrm{K}}_{\phi}(e) \wedge \tilde{\mathrm{K}}_{\phi}(f)\right\} \wedge\left\{\tilde{\mathrm{K}}_{\psi}(e) \wedge \tilde{\mathrm{K}}_{\psi}(f)\right\} \\
& =\left\{\tilde{\mathrm{K}}_{\phi}(e) \wedge \tilde{\mathrm{K}}_{\psi}(e)\right\} \wedge\left\{\tilde{\mathrm{K}}_{\phi}(f) \wedge \tilde{\mathrm{K}}_{\psi}(f)\right\} \\
& =\left(\tilde{\mathrm{K}}_{\phi} \cap \tilde{\mathrm{K}}_{\psi}\right)(e) \wedge\left(\tilde{\mathrm{K}}_{\phi} \cap \tilde{\mathrm{K}}_{\psi}\right)(f) .
\end{aligned}
$$

(2) Now,

$$
\begin{aligned}
\left(\tilde{\mathrm{K}}_{\phi} \cap \tilde{\mathrm{K}}_{\psi}\right)(e f) & =\tilde{\mathrm{K}}_{\phi}(e f) \wedge \tilde{\mathrm{K}}_{\psi}(e f) \geq \tilde{\mathrm{K}}_{\phi}(f) \wedge \tilde{\mathrm{K}}_{\psi}(f) \\
& =\left(\tilde{\mathrm{K}}_{\phi} \cap \tilde{\mathrm{K}}_{\psi}\right)(f) .
\end{aligned}
$$

(3) Let $e+x=y$, then

$$
\begin{aligned}
\left(\tilde{\mathrm{K}}_{\phi} \cap \tilde{\mathrm{K}}_{\psi}\right)(e) & \geq\left\{\left(\tilde{\mathrm{K}}_{\phi}(x)\right) \wedge \tilde{\mathrm{K}}_{\phi}(y)\right\} \wedge\left\{\tilde{\mathrm{K}}_{\psi}(x) \wedge \tilde{\mathrm{K}}_{\psi}(y)\right\} \\
& =\left\{\tilde{\mathrm{K}}_{\phi}(x) \wedge \tilde{\mathrm{K}}_{\psi}(x) \wedge \tilde{\mathrm{K}}_{\phi}(y) \wedge \tilde{\mathrm{K}}_{\psi}(y)\right\} \\
& =\left(\tilde{\mathrm{K}}_{\phi} \cap \tilde{\mathrm{K}}_{\psi}\right)(x) \wedge\left(\tilde{\mathrm{K}}_{\phi} \cap \tilde{\mathrm{K}}_{\psi}\right)(y) .
\end{aligned}
$$

For right $k$-ideals, the proof is similar.

Theorem 5. If the falling fuzzy set $\tilde{K}$ is the falling fuzzy left (right) $k$-ideal on $T$, then

$$
\chi_{T} \bigodot_{I} \tilde{K} \subseteq \tilde{K}\left(\tilde{K} \bigodot_{I} \chi_{T} \subseteq \tilde{K}\right) .
$$

Proof. Suppose $\tilde{\mathrm{K}}$ is a falling fuzzy $k$-ideal on $T$, let $e \in T$. If
$\left(\chi_{T} \bigodot_{I} \tilde{\mathrm{K}}\right)(e)=0$, then $\left(\chi_{T} \bigodot_{I} \tilde{\mathrm{K}}\right)(e) \tilde{\mathrm{K}}(e)$. Otherwise, there are $s_{r}, t_{r}, \hat{s}_{m}, \hat{t}_{m} \in T$ such that $e+\sum_{r=1}^{u} s_{r} t_{r}=\sum_{m=1}^{v} \dot{s}_{m} \hat{t}_{m}$, so

$$
\begin{aligned}
& \left(\chi_{T} \bigodot_{I} \tilde{\mathrm{K}}\right)(e)=\bigvee_{e+\sum_{r=1}^{u} s_{r} t_{r}=\sum_{m=1}^{v} s_{m} t_{m}}\left\{\tilde{\mathrm{K}}\left(t_{r}\right) \wedge \tilde{\mathrm{K}}\left(\tilde{t}_{m}\right)\right\} \\
& \leq \quad \vee \\
& e+\sum_{r=1}^{u} s_{r} t_{r}=\sum_{m=1}^{v} s_{m} t_{m}\left\{\tilde{\mathrm{K}}\left(s_{r} t_{r}\right) \wedge \tilde{\mathrm{K}}\left(\hat{s}_{m} t_{m}\right)\right\} \\
& \leq \quad V \\
& e+\sum_{r=1}^{u} s_{r} t_{r}=\sum_{m=1}^{v} \hat{s}_{m} \hat{t}_{m}\left\{\tilde{\mathrm{K}}\left(\sum_{r=1}^{u} s_{r} t_{r}\right) \wedge \tilde{\mathrm{K}}\left(\sum_{m=1}^{v} \dot{s}_{m} \hat{t}_{m}\right)\right\} \\
& \leq \quad \mathrm{V} \\
& e+\sum_{r=1}^{u} s_{r} t_{r}=\sum_{m=1}^{v} s_{m} t_{m} \tilde{\mathrm{K}}(e)=\tilde{\mathrm{K}}(e) .
\end{aligned}
$$

It implies that $\chi_{T} \bigodot_{I} \tilde{\mathrm{K}} \subseteq \tilde{\mathrm{K}}$.

The proof is similar for falling fuzzy right $k$-ideals.

Theorem 6. Let $T$ be a hemiring and $X, Y \subseteq T$, then $\left(\chi_{X} \bigodot_{I}\right.$ $\left.\chi_{Y}\right)=\chi_{X Y}$.

Proof. If $e \in \overline{X Y}$, then $\chi_{X Y}(e)=1$ and $e+\sum_{r=1}^{u} s_{r} t_{r}=\sum_{m=1}^{v}$ $\dot{s}_{m} \hat{t}_{m}$ for all $s_{r}, \dot{s}_{m} \in X, t_{r}, \hat{t}_{m} \in Y$. Let $e \in T$. We have

$$
\begin{aligned}
\left(\chi_{X} \bigodot_{I} \chi_{Y}\right)(e) & =\underset{e+\sum_{r=1}^{u} s_{r} t_{r}=\sum_{m=1}^{v} s_{m} \dot{t}_{m}\left\{\chi_{X}\left(s_{r}\right) \wedge \chi_{X}\left(\dot{s}_{m}\right) \wedge \chi_{Y}\left(t_{r}\right) \wedge \chi_{Y}\left(\dot{t}_{m}\right)\right\}}{\bigvee} \\
& \geq \chi_{X}\left(s_{r}\right) \wedge \chi_{X}\left(\dot{s}_{m}\right) \wedge \chi_{Y}\left(t_{r}\right) \wedge \chi_{Y}\left(\dot{t}_{m}\right)=1 .
\end{aligned}
$$

That is, $\left(\chi_{X} \bigodot_{I} \chi_{Y}\right)(e)=1=\chi_{X Y}(e)$. Hence, $\left(\chi_{X} \bigodot_{I} \chi_{Y}\right)$ $=\chi_{X \bar{Y}}$. If $e \notin \overline{X Y}$, then $\chi_{X \bar{Y}}(e)=0$. Let $\left(\chi_{X} \bigodot_{I} \chi_{Y}\right) \neq 0$, and then

$\left(\chi_{X} \bigodot_{I} \chi_{Y}\right)(e)=\underset{e+\sum_{r=1}^{u} s_{r} t_{r}=\sum_{m=1}^{v} \hat{s}_{m} \hat{t}_{m}}{\hat{t}_{m}}\left\{\chi_{X}\left(s_{r}\right) \wedge \chi_{X}\left(\hat{s}_{m}\right) \wedge \chi_{Y}\left(t_{r}\right) \wedge \chi_{Y}\left(\hat{t}_{m}\right)\right\} \neq 0$.

Hence, there are $s_{r}, \dot{s}_{m}, t_{r}, \hat{t}_{m} \in T$ such that $e+\sum_{r=1}^{u} s_{r} t_{r}$ $=\sum_{m=1}^{v} \dot{s}_{m} \dot{t}_{m}$ and

$$
\chi_{X}\left(s_{r}\right) \wedge \chi_{X}\left(s_{m}\right) \wedge \chi_{Y}\left(t_{r}\right) \wedge \chi_{Y}\left(\dot{t}_{m}\right) \neq 0
$$

imply that

$$
\chi_{X}\left(s_{r}\right)=\chi_{X}\left(\dot{s}_{m}\right)=\chi_{Y}\left(t_{r}\right)=\chi_{Y}\left(\dot{t}_{m}\right)=1
$$

for all $s_{r}, \dot{s}_{m} \in X, t_{r}, t_{m} \in Y$ and $e \in \overline{X Y}$, which is a contradiction to $\chi_{X Y}(e)=0$. So, our supposition is wrong, and $\left(\chi_{X}\right.$ $\left.\bigodot_{I} \chi_{Y}\right)(e)=0=\chi_{X Y}(e)$.

In both cases,

$$
\left(\chi_{X} \bigodot_{I} \chi_{Y}\right)=\chi_{X Y}
$$

which completes the proof. 
Theorem 7. A hemiring $T$ is a $k$-hemiregular if for any falling fuzzy right $k$-ideal $\tilde{K}_{\phi}$ and any falling fuzzy left $k$-ideal $\tilde{K}_{\psi}$ of $T$, we have

$$
\tilde{K}_{\phi} \bigodot_{I} \tilde{K}_{\psi}=\tilde{K}_{\phi} \cap \tilde{K}_{\psi} .
$$

Proof. Suppose $T$ is a $k$-hemiregular hemiring, let $\tilde{\mathrm{K}}_{\phi}$ (resp., $\tilde{\mathrm{K}}_{\psi}$ ) be a falling fuzzy right (resp., left) $k$-ideal of $T$. Then,

$$
\begin{gathered}
\tilde{\mathrm{K}}_{\phi} \bigodot_{I} \tilde{\mathrm{K}}_{\psi} \subseteq \tilde{\mathrm{K}}_{\phi} \bigodot_{I} \chi_{T} \subseteq \tilde{\mathrm{K}}_{\phi}, \\
\tilde{\mathrm{K}}_{\phi} \bigodot_{I} \tilde{\mathrm{K}}_{\psi} \subseteq \chi_{T} \bigodot_{I} \tilde{\mathrm{K}}_{\psi} \subseteq \tilde{\mathrm{K}}_{\psi} \text { Then } \tilde{\mathrm{K}}_{\phi} \bigodot_{I} \tilde{\mathrm{K}}_{\psi} \subseteq \tilde{\mathrm{K}}_{\phi} \cap \tilde{\mathrm{K}}_{\psi} .
\end{gathered}
$$

Conversely, suppose $e \in T$, as $T$ is a $k$-hemiregular, so there are $x, \dot{x} \in T$ such that

$$
\begin{aligned}
e+e x e= & e x e,\left(\tilde{\mathrm{K}}_{\phi} \bigodot_{I} \tilde{\mathrm{K}}_{\psi}\right)(e) \\
= & \quad \vee+\sum_{r=1}^{u} s_{r} t_{r}=\sum_{m=1}^{v} \dot{s}_{m} \dot{t}_{m}\left\{\tilde{\mathrm{K}}_{\phi}\left(s_{r}\right) \wedge \tilde{\mathrm{K}}_{\phi}\left(\tilde{s}_{m}\right) \wedge \tilde{\mathrm{K}}_{\psi}\left(t_{r}\right) \wedge \tilde{\mathrm{K}}_{\psi}\left(\hat{t}_{m}\right)\right\} \\
\geq & \tilde{\mathrm{K}}_{\phi}(e x) \wedge \tilde{\mathrm{K}}_{\phi}(\bar{e} \dot{x}) \wedge \tilde{\mathrm{K}}_{\psi}(e) \geq \tilde{\mathrm{K}}_{\phi}(e) \wedge \tilde{\mathrm{K}}_{\psi}(e) \\
= & \left(\tilde{\mathrm{K}}_{\phi} \cap \tilde{\mathrm{K}}_{\psi}\right)(e) .
\end{aligned}
$$

This implies that

$$
\tilde{\mathrm{K}}_{\phi} \bigodot_{I} \tilde{\mathrm{K}}_{\psi} \supseteq \tilde{\mathrm{K}}_{\phi} \cap \tilde{\mathrm{K}}_{\psi} .
$$

Therefore,

$$
\tilde{\mathrm{K}}_{\phi} \bigodot_{I} \tilde{\mathrm{K}}_{\psi}=\tilde{\mathrm{K}}_{\phi} \cap \tilde{\mathrm{K}}_{\psi} .
$$

Conversely, suppose $\zeta_{\phi}(\mathrm{q})$ (resp., $\zeta_{\psi}(\mathrm{q})$ is a left $k$-ideal (resp., right) $k$-ideal of $T$, then $\tilde{\mathrm{K}}_{\phi}(v)$ (resp., $\tilde{\mathrm{K}}_{\psi}(v)$ ) is a falling fuzzy left (resp., right) $k$-ideal of $T$. So, $\chi_{\zeta_{\phi}(\mathrm{q})}\left(\right.$ resp., $\chi_{\zeta_{\psi}(\mathrm{q})}$ ) is the characteristic function of the fuzzy left (resp., right) $k$ -ideal of $T$. We have

$$
\begin{aligned}
& \chi_{\zeta_{\phi}(\mathrm{q}) \bar{\zeta}_{\psi}(\mathrm{q})}=\chi_{\zeta_{\phi}(\mathrm{q})} \bigodot_{I} \chi_{\zeta_{\psi}(\mathrm{q})}=\chi_{\zeta_{\phi}(\mathrm{q})} \cap \chi_{\zeta_{\psi}(\mathrm{q})}=\chi_{\zeta_{\phi}(\mathrm{q}) \cap \zeta_{\psi}(\mathrm{q})}, \\
& \zeta_{\phi}(\mathrm{q}) \bar{\zeta}_{\psi}(\mathrm{q})=\zeta_{\phi}(\mathrm{q}) \cap \zeta_{\psi}(\mathrm{q}) .
\end{aligned}
$$

Thus, we have

$\left(\tilde{\mathrm{K}}_{\phi} \tilde{\mathrm{K}}_{\psi}\right)(v)=R\left(\mathrm{q} \mid v \in \zeta_{\phi}(\mathrm{q}) \bar{\zeta}_{\psi}(\mathrm{q})\right)=R\left(\mathrm{q} \mid v \in \zeta_{\phi}(\mathrm{q}) \cap \zeta_{\psi}(\mathrm{q})\right)=\left(\tilde{\mathrm{K}}_{\phi} \cap \tilde{\mathrm{K}}_{\psi}\right)(v)$.
Definition 15 [29]. Let $\Lambda$ be a probability space, and then $\Lambda$ is called an independent probability space if

$$
\Lambda(e, \zeta) \cap \Lambda(f, \zeta)=\Lambda(e, \zeta) \Lambda(f, \zeta), \forall e, f \in T .
$$

Definition 16. Let $\Lambda$ be an IPS, and suppose that $\tilde{K}_{\phi}$ and $\tilde{K}_{\psi}$ are falling fuzzy $k$-ideals on $T$, then the $L$-product of $\tilde{K}_{\phi}$ and $\tilde{K}_{\psi}$ is defined by

$$
\left(\tilde{\mathrm{K}}_{\phi} \bigodot_{L} \tilde{\mathrm{K}}_{\psi}\right)(e)=\left\{\tilde{\mathrm{K}}_{\phi}\left(s_{r}\right) \tilde{\mathrm{K}}_{\phi}\left(\dot{s}_{m}\right) \tilde{\mathrm{K}}_{\psi}\left(t_{r}\right) \tilde{\mathrm{K}}_{\psi}\left(\dot{t}_{m}\right), \text { if } e+\sum_{r=1}^{u} s_{r} t_{r}=\sum_{m=1}^{v} s_{m}^{\prime} t_{m}^{\prime} 0\right. \text {, otherwise. }
$$

Theorem 8. Let $\Lambda$ be an IPS and $\tilde{K}$ be a falling fuzzy left (right) $k$-ideal of $T$ for all $e, f, x, y \in T$. Then,

(1) $\tilde{K}(e+f) \geq \tilde{K}(e) \tilde{K}(f)$

(2) $\tilde{K}(e f) \geq \tilde{K}(f)(\tilde{K}(e f) \geq \tilde{K}(e))$

(3) $e+x=y \Rightarrow \tilde{K}(e) \geq \tilde{K}(x) \tilde{K}(y)$

Proof.

(1) Let $\zeta$ (q) be a left $k$-ideal on $T$ for any $q \in \Lambda$. As we know that $\Lambda(\mathrm{e}, \zeta) \cap \Lambda(\mathrm{f}, \zeta) \subseteq \Lambda(\mathrm{e}+\mathrm{f}, \zeta)$, then

$$
\begin{aligned}
\tilde{\mathrm{K}}(e+f) & =R(\mathrm{q} \mid e+\mathrm{f} \in \zeta(\mathrm{q})) \geq R(\{\mathrm{q} \mid e \in \zeta(\mathrm{q})\} \cap\{\mathrm{q} \mid f \in \zeta(\mathrm{q})\}) \\
& =R(\{\mathrm{q} \mid e \in \zeta(\mathrm{q})\}) R(\{\mathrm{q} \mid f \in \zeta(\mathrm{q})\})=\tilde{\mathrm{K}}(e) \tilde{\mathrm{K}}(f)
\end{aligned}
$$

(2) Let $\zeta(q)$ be a left $k$-ideal of $T$ for any $q \in \Lambda$. As $\Lambda(f$ $, \zeta) \subseteq \Lambda(e f, \zeta)$, so we have

$$
\tilde{\mathrm{K}}(e f)=R(\mathrm{q} \mid e f \in \zeta(\mathrm{q})) \geq R(\mathrm{q} \mid f \in \zeta(\mathrm{q}))=\tilde{\mathrm{K}}(f)
$$

(3) Let $\zeta$ (q) be a left $k$-ideal of $T$ for any $\mathrm{q} \in \Lambda$. So,

$$
e+x=y \Rightarrow \Lambda(x, \zeta) \cap \Lambda(y, \zeta) \subseteq \Lambda(e, \zeta)
$$

Hence,

$$
\begin{aligned}
\tilde{\mathrm{K}}(e) & =R(\mathrm{q} \mid e \in \zeta(\mathrm{q})) \geq R(\{\mathrm{q} \mid x \in \zeta(\mathrm{q})\} \cap\{\mathrm{q} \mid y \in \zeta(\mathrm{q})\}) \\
& =\mathrm{R}(\{\mathrm{q} \mid x \in \zeta(\mathrm{q})\}) R(\{\mathrm{q} \mid y \in \zeta(\mathrm{q})\})
\end{aligned}
$$

$$
\tilde{\mathrm{K}}(e)=\tilde{\mathrm{K}}(x) \tilde{\mathrm{K}}(y) .
$$

Hence, $T$ is a $k$-hemiregular hemiring. 
TABLE 1

\begin{tabular}{lllll}
\hline+ & 0 & $e^{b}$ & $f^{b}$ & $g^{b}$ \\
0 & 0 & $e^{b}$ & $f^{b}$ & $g^{b}$ \\
$e^{b}$ & $e^{b}$ & $e^{b}$ & $f^{b}$ & $g^{b}$ \\
$f^{b}$ & $f^{b}$ & $f^{b}$ & $f^{b}$ & $g^{b}$ \\
$g^{b}$ & $g^{b}$ & $g^{b}$ & $g^{b}$ & $f^{b}$ \\
$\cdot$ & 0 & $e^{b}$ & $f^{b}$ & $g^{b}$ \\
0 & 0 & 0 & 0 & 0 \\
$e^{b}$ & 0 & $e^{b}$ & $e^{b}$ & $e^{b}$ \\
$f^{b}$ & 0 & $e^{b}$ & $e^{b}$ & $e^{b}$ \\
$g^{b}$ & 0 & $e^{b}$ & $e^{b}$ & $e^{b}$ \\
\hline
\end{tabular}

TABLE 2

\begin{tabular}{cccccc}
\hline+ & 0 & $e^{b}$ & $x^{b}$ & $y^{b}$ & $z^{b}$ \\
0 & 0 & $e^{b}$ & $x^{b}$ & $y^{b}$ & $z^{b}$ \\
$e^{b}$ & $e^{b}$ & $y^{b}$ & $e^{b}$ & $x^{b}$ & $e^{b}$ \\
$x^{b}$ & $f^{b}$ & $f^{b}$ & $f^{b}$ & $g^{b}$ & $x^{b}$ \\
$y^{b}$ & $g^{b}$ & $g^{b}$ & $g^{b}$ & $f^{b}$ & $y^{b}$ \\
$z^{b}$ & $z^{b}$ & $e^{b}$ & $x^{b}$ & $y^{b}$ & $z^{b}$ \\
$\cdot$ & 0 & $e^{b}$ & $x^{b}$ & $y^{b}$ & $z^{b}$ \\
0 & 0 & 0 & 0 & 0 & 0 \\
$e^{b}$ & 0 & $e^{b}$ & $e^{b}$ & $z^{b}$ & $z^{b}$ \\
$x^{b}$ & 0 & $e^{b}$ & $e^{b}$ & 0 & $z^{b}$ \\
$y^{b}$ & 0 & $y^{b}$ & $x^{b}$ & $e^{b}$ & $z^{b}$ \\
$z^{b}$ & 0 & $z^{b}$ & $z^{b}$ & $z^{b}$ & 0 \\
\hline
\end{tabular}

Proposition 4 [27]. If $\Lambda$ is an independent probability space, then

$$
\left(\tilde{K}_{\phi} \cap \tilde{K}_{\psi}\right)(e)=\tilde{K}_{\phi}(e) \tilde{K}_{\psi}(e) .
$$

Proposition 5. Let $\Lambda$ be an independent probability space and $\tilde{K}$ be a falling fuzzy $k$-ideal on $T$, and then

$$
\tilde{K}(e f) \geq \tilde{K}(e)+\tilde{K}(f)-\tilde{K}(e) \tilde{K}(f) .
$$

Proof. Since $\Lambda(e f, \zeta) \supseteq \Lambda(e, \zeta) \cap \Lambda(f, \zeta)$, one gets

$$
\begin{aligned}
\tilde{\mathrm{K}}(e f) & =R(\mathrm{q} \mid e f \in \zeta(\mathrm{q})) \geq R(\{\mathrm{q} \mid e \in \zeta(\mathrm{q})\} \cap\{\mathrm{q} \mid f \in \zeta(\mathrm{q})\}) \\
& \geq R(\mathrm{q} \mid e \in \zeta(\mathrm{q}))+R(\mathrm{q} \mid f \in \zeta(\mathrm{q}))-R((\mathrm{q} \mid e \in \zeta(\mathrm{q})) \cap(\mathrm{q} \mid f \in \zeta(\mathrm{q}))) \\
& =R(\mathrm{q} \mid e \in \zeta(\mathrm{q}))+R(\mathrm{q} \mid f \in \zeta(\mathrm{q}))-R((\mathrm{q} \mid e \in \zeta(\mathrm{q})) R(\mathrm{q} \mid f \in \zeta(\mathrm{q}))) \\
& \geq \tilde{\mathrm{K}}(e)+\tilde{\mathrm{K}}(f)-\tilde{\mathrm{K}}(e) \tilde{\mathrm{K}}(f) .
\end{aligned}
$$

TABLE 3

\begin{tabular}{ccccc}
\hline+ & 0 & $x^{b}$ & $y^{b}$ & $z^{b}$ \\
0 & 0 & $x^{b}$ & $y^{b}$ & $z^{b}$ \\
$x^{b}$ & $x^{b}$ & 0 & $z^{b}$ & $y^{b}$ \\
$y^{b}$ & $y^{b}$ & $z^{b}$ & 0 & $x^{b}$ \\
$z^{b}$ & $z^{b}$ & $y^{b}$ & $x^{b}$ & 0 \\
$\cdot$ & 0 & $x^{b}$ & $y^{b}$ & $z^{b}$ \\
0 & 0 & 0 & 0 & 0 \\
$x^{b}$ & 0 & 0 & 0 & 0 \\
$y^{b}$ & 0 & 0 & $z^{b}$ & $z^{b}$ \\
$z^{b}$ & 0 & 0 & $z^{b}$ & $z^{b}$ \\
\hline
\end{tabular}

Proposition 6. Suppose $\Lambda$ is an IPS, let $\tilde{K}_{\phi}$ and $\tilde{K}_{\psi}$ be falling fuzzy left (right) $k$-ideals of $T$, and then $\tilde{K}_{\phi} \cap \tilde{K}_{\psi}$ is also a falling fuzzy left (right) $k$-ideal of $T$.

Proof. Let $\tilde{\mathrm{K}}_{\phi}$ and $\tilde{\mathrm{K}}_{\psi}$ be falling fuzzy left $k$-ideals of $T$, and then

$$
\begin{aligned}
& \left(\tilde{\mathrm{K}}_{\phi} \cap \tilde{\mathrm{K}}_{\psi}\right)(e+f)=\tilde{\mathrm{K}}_{\phi}(e+f) \tilde{\mathrm{K}}_{\psi}(e+f) \geq \tilde{\mathrm{K}}_{\phi}(e) \tilde{\mathrm{K}}_{\phi}(f) \tilde{\mathrm{K}}_{\psi}(e) \tilde{\mathrm{K}}_{\psi}(f) \\
& =\left(\tilde{\mathrm{K}}_{\phi} \cap \tilde{\mathrm{K}}_{\psi}\right)(e)\left(\tilde{\mathrm{K}}_{\phi} \cap \tilde{\mathrm{K}}_{\psi}\right)(f) \text {, } \\
& \left(\tilde{\mathrm{K}}_{\phi} \cap \tilde{\mathrm{K}}_{\psi}\right)(e f)=\tilde{\mathrm{K}}_{\phi}(e f) \tilde{\mathrm{K}}_{\psi}(e f) \geq \tilde{\mathrm{K}}_{\phi}(f) \tilde{\mathrm{K}}_{\psi}(f)=\left(\tilde{\mathrm{K}}_{\phi} \cap \tilde{\mathrm{K}}_{\psi}\right)(f) .
\end{aligned}
$$

(1) Let $\mathrm{e}+x=y$, and then

$\left(\tilde{\mathrm{K}}_{\phi} \cap \tilde{\mathrm{K}}_{\psi}\right)(e) \geq \tilde{\mathrm{K}}_{\phi}(x) \tilde{\mathrm{K}}_{\phi}(y) \tilde{\mathrm{K}}_{\psi}(x) \tilde{\mathrm{K}}_{\psi}(y)=\left(\tilde{\mathrm{K}}_{\phi} \cap \tilde{\mathrm{K}}_{\psi}\right)(x)\left(\tilde{\mathrm{K}}_{\phi} \cap \tilde{\mathrm{K}}_{\psi}\right)(y)$.

For the case of right $k$-ideals, the proof is found on similar lines.

Theorem 9. If the falling fuzzy set $\tilde{K}$ of $T$ is a falling fuzzy left (right) $k$-ideal of $T$, then

$$
\chi_{T} \bigodot_{L} \tilde{K} \subseteq \tilde{K}\left(\tilde{K} \bigodot_{L} \chi_{T} \subseteq \tilde{K}\right) .
$$

Proof. Suppose $\tilde{K}$ is a falling fuzzy $k$-ideal of $T$ and $e \in T$, if $\left(\chi_{T} \bigodot_{L} \tilde{\mathrm{K}}\right)(e)=0$, then $\left(\chi_{T} \bigodot_{L} \tilde{\mathrm{K}}\right)(e) \tilde{\mathrm{K}}(e)$. Otherwise, there are $s_{r}, t_{r}, \hat{s}_{m}, \hat{t}_{m} \in T$ such that $e+\sum_{r=1}^{u} s_{r} t_{r}=\sum_{m=1}^{v} \dot{s}_{m} t_{m}$; so,

$$
\left(\chi_{T} \bigodot_{L} \tilde{\mathrm{K}}\right)(e)=\tilde{\mathrm{K}}\left(t_{r}\right) \tilde{\mathrm{K}}\left(\dot{t}_{m}\right) \tilde{\mathrm{K}}\left(s_{r} t_{r}\right) \tilde{\mathrm{K}}\left(\dot{s}_{m} \dot{t}_{m}\right) \tilde{\mathrm{K}}\left(\sum_{r=1}^{u} s_{r} t_{r}\right) \tilde{\mathrm{K}}\left(\sum_{m=1}^{v} \dot{s}_{m} \dot{t}_{m}\right) \tilde{\mathrm{K}}(e) .
$$

Thus, $\chi_{T} \bigodot_{L} \tilde{\mathrm{K}} \subseteq \tilde{\mathrm{K}}$. 
The proof is similar for right $k$-ideals.

Theorem 10. If $T$ is a hemiring and $X, Y \subseteq T$, then $\chi_{X} e_{L} \chi_{Y}$ $=\chi_{X Y}$.

Proof. Let $e \in T$. If $e \in \overline{X Y}$, then $\chi_{X Y}(e)=1$ and $e+\sum_{r=1}^{u} s_{r} t_{r}$ $=\sum_{m=1}^{v} \dot{s}_{m} \dot{t}_{m}$, for all $s_{r}, \dot{s}_{m} \in X, t_{r}, \dot{t}_{m} \in Y$. So, we have

$$
\begin{aligned}
\left(\chi_{X} \bigodot_{L} \chi_{Y}\right)(e) & =\chi_{X}\left(s_{r}\right) \chi_{X}\left(\dot{s}_{m}\right) \chi_{Y}\left(t_{r}\right) \chi_{Y}\left(\dot{t}_{m}\right) \\
& \geq \chi_{X}\left(s_{r}\right) \chi_{X}\left(\dot{s}_{m}\right) \chi_{Y}\left(t_{r}\right) \chi_{Y}\left(\dot{t}_{m}\right)=1
\end{aligned}
$$

Thus, $\left(\chi_{X} \bigodot_{L} \chi_{Y}\right)(e)=1=\chi_{X Y}(e)$. If $e \notin \overline{X Y}$, then $\chi_{X Y}$ $(e)=0$. Let us suppose that $\left(\chi_{X} \bigodot_{L} \chi_{Y}\right) \neq 0$, and then

$$
\left(\chi_{X} \bigodot_{L} \chi_{Y}\right)(e)=\chi_{X}\left(s_{r}\right) \chi_{X}\left(\dot{s}_{m}\right) \chi_{Y}\left(t_{r}\right) \chi_{Y}\left(\dot{t}_{m}\right) \neq 0
$$

Therefore, there are $s_{r}, \dot{s}_{m}, t_{r}, t_{m} \in T$ such that $e+\sum_{r=1}^{u} s_{r}$ $t_{r}=\sum_{m=1}^{v} s_{m} t_{m}$ and

$$
\chi_{X}\left(s_{r}\right) \chi_{X}\left(\dot{s}_{m}\right) \chi_{Y}\left(t_{r}\right) \chi_{Y}\left(\dot{t}_{m}\right) \neq 0, \chi_{X}\left(s_{r}\right)=\chi_{X}\left(\dot{s}_{m}\right)=\chi_{Y}\left(t_{r}\right)=\chi_{Y}\left(\dot{t}_{m}\right)=1 .
$$

Hence, for all $s_{r}, \dot{s}_{m} \in X, t_{r}, t_{m} \in Y$ and $e \in \overline{X Y}$, which is a contradiction to $\chi_{X Y}(e)=0$, our supposition is wrong, and

$$
\left(\chi_{X} \bigodot_{L} \chi_{Y}\right)(e)=0=\chi_{X Y}(e)
$$

This implies that

$$
\left(\chi_{X} \bigodot_{L} \chi_{Y}\right)(e)=\chi_{X Y}(e)
$$

Theorem 11. A hemiring $T$ is a $k$-hemiregular if for any falling fuzzy right $k$-ideal $\tilde{K}_{\phi}$ of $T$ and any falling fuzzy left $k$ -ideal $\tilde{K}_{\psi}$ on $T$, we have $\tilde{K}_{\phi} e_{L} \tilde{K}_{\psi}=\tilde{K}_{\phi} \cap \tilde{K}_{\psi}$.

Proof. Suppose $T$ is a $k$-hemiregular hemiring, let $\tilde{K}_{\phi}$ (resp., $\tilde{K}_{\psi}$ ) be the falling fuzzy right (resp., left) $k$-ideal of $T$. Then,

$$
\begin{aligned}
& \tilde{\mathrm{K}}_{\phi} \bigodot_{L} \tilde{\mathrm{K}}_{\psi} \subseteq \tilde{\mathrm{K}}_{\phi} \mathrm{e}_{L} \chi_{T} \subseteq \tilde{\mathrm{K}}_{\phi}, \\
& \tilde{\mathrm{K}}_{\phi} \bigodot_{L} \tilde{\mathrm{K}}_{\psi} \subseteq \chi_{T} \mathrm{e}_{L} \tilde{\mathrm{K}}_{\phi} \subseteq \tilde{\mathrm{K}}_{\psi} .
\end{aligned}
$$

It implies that

$$
\tilde{\mathrm{K}}_{\phi} \bigodot_{L} \tilde{\mathrm{K}}_{\psi} \subseteq \tilde{\mathrm{K}}_{\phi} \cap \tilde{\mathrm{K}}_{\psi}
$$

Suppose $e \in T$, since $T$ is a $k$-hemiregular, there are $x$, $\dot{x} \in T$ such that $e+$ exe $=$ ex e where $e+\sum_{r=1}^{u} s_{r} t_{r}=\sum_{m=1}^{v} \dot{s}_{m} t_{m}$, for all $s_{r}, \dot{s}_{m}, t_{r}, \dot{t}_{m} \in T$. We have

$$
\begin{aligned}
\left(\tilde{\mathrm{K}}_{\phi} \odot{ }_{L} \tilde{\mathrm{K}}_{\psi}\right)(e) & =\tilde{\mathrm{K}}_{\phi}\left(s_{r}\right) \tilde{\mathrm{K}}_{\phi}\left(\dot{s}_{m}\right) \tilde{\mathrm{K}}_{\psi}\left(t_{r}\right) \tilde{\mathrm{K}}_{\psi}\left(\dot{t}_{m}\right) \\
& \geq \tilde{\mathrm{K}}_{\phi}(e x e) \tilde{\mathrm{K}}_{\phi}(e x e) \geq \tilde{\mathrm{K}}_{\phi}(x e) \tilde{\mathrm{K}}_{\psi}(\text { ex } e) \\
& \geq \tilde{\mathrm{K}}_{\phi}(e) \tilde{\mathrm{K}}_{\psi}(e)=\left(\tilde{\mathrm{K}}_{\phi} \cap \tilde{\mathrm{K}}_{\psi}\right)(e) .
\end{aligned}
$$

It yields that $\tilde{\mathrm{K}}_{\phi} \bigodot_{L} \tilde{\mathrm{K}}_{\psi} \supseteq \tilde{\mathrm{K}}_{\phi} \cap \tilde{\mathrm{K}}_{\psi}$.

Conversely, suppose that $\zeta_{\phi}(\mathrm{q})$ and $\zeta_{\psi}(\mathrm{q})$ are right $k$ -ideal and left $k$-ideal of $T$, respectively, then $\tilde{K}_{\phi}(\bar{v})$ and $\tilde{K}_{\psi}$ $(\bar{v})$ are both a falling fuzzy right $k$-ideal and a falling fuzzy left $k$-ideal of $T$. So, $\chi_{\zeta_{\phi}(\mathrm{q})}$ and $\chi_{\zeta_{\psi}(\mathrm{q})}$. Hence, the characteristic function of $\zeta_{\phi}(\mathrm{q})$ and $\zeta_{\psi}(\mathrm{q})$ is a fuzzy right $k$-ideal and a fuzzy left $k$-ideal of $T$, respectively. We have

$$
\begin{aligned}
\bar{v}_{\zeta_{\phi}(\mathrm{q}) \bar{\zeta}_{\psi}(\mathrm{q})=}=\bar{v}_{\zeta_{\phi}(\mathrm{q})} \bigodot_{L} \bar{v}_{\zeta_{\psi}(\mathrm{q})}=\bar{v}_{\zeta_{\phi}(\mathrm{q})} \cap \bar{v}_{\zeta_{\psi}(\mathrm{q})}=\bar{v}_{\zeta_{\phi}(\mathrm{q}) \cap \zeta_{\psi}(\mathrm{q})}, \\
\zeta_{\phi}(\mathrm{q}) \bar{\zeta}_{\psi}(\mathrm{q})=\zeta_{\phi}(\mathrm{q}) \cap \zeta_{\psi}(\mathrm{q}) .
\end{aligned}
$$

Thus,

$$
\begin{aligned}
\left(\tilde{\mathrm{K}}_{\phi} \tilde{\mathrm{K}}_{\psi}\right)(\bar{v}) & =R\left(\mathrm{q} \mid \bar{v} \in \zeta_{\phi}(\mathrm{q}) \bar{\zeta}_{\psi}(\mathrm{q})\right)=R\left(\mathrm{q} \mid \bar{v} \in \zeta_{\phi}(\mathrm{q}) \cap \zeta_{\psi}(\mathrm{q})\right) \\
& =\left(\tilde{\mathrm{K}}_{\phi} \cap \tilde{\mathrm{K}}_{\psi}\right)(\bar{v}) .
\end{aligned}
$$

Hence, $T$ is a $k$-hemiregular hemiring.

\section{Conclusion}

In this paper, we introduced the notion of falling fuzzy $k$ -ideals of a hemiring and illustrated it with the help of examples. Then, we investigated some characteristics of $k$-hemiregulars by means of falling fuzzy $k$-ideals based on a perfect positive correlation and an independent probability space. In future works, one can consider $k$-hemiregulars using falling fuzzy $k$-bi-ideals and falling fuzzy $k$-quasiideals. One also can apply fuzzy inference relations to hemirings. Further, one can investigate this theory to information science, intelligent and fuzzy systems, etc. Moreover, the idea of falling fuzzy $k$-ideals can be extended to BCI-algebras [4] and KU-Algebras [5].

\section{Data Availability}

The data used to support the findings of this study are available from the corresponding author upon request.

\section{Conflicts of Interest}

The authors declare that they have no competing interests. 


\section{Authors' Contributions}

All authors contributed equally and significantly in writing this article. All authors read and approved the final manuscript.

\section{Acknowledgments}

The fourth author would like to acknowledge that his contribution to this work was carried out with the aid of a grant from the Carnegie Corporation provided through the African Institute for Mathematical Sciences.

\section{References}

[1] H. S. Vandiver, "Note on a simple type of algebra in which the cancellation law of addition does not hold," Bulletin of the American Mathematical Society, vol. 40, no. 12, pp. 914-921, 1934.

[2] J. E. Hopcroft, R. Motwani, and J. D. Ullman, "Introduction to automata theory, languages, and computation, 2nd edition," ACM SIGACT News, vol. 32, no. 1, pp. 60-65, 2001.

[3] D. B. Benson, Bialgebras: Some Foundations for Distributed and Concurrent Computation, Computer Science Department, Washington State University, 1987.

[4] T. Senapati, Y. B. Jun, G. Muhiuddin, and K. P. Shum, "Cubic intuitionistic structures applied to ideals ofBCI-algebras," Analele Universitatii" Ovidius" Constanta-Seria Matematica, vol. 27, no. 2, pp. 213-232, 2019.

[5] T. Senapati and K. P. Shum, "Atanassov's intuitionistic fuzzy bi-normed KU-subalgebras of a KU-algebra," Missouri Journal of Mathematical Sciences, vol. 29, no. 1, pp. 92-112, 2017.

[6] M. Munir, "On m-bi ideals in semigroups," Bulletin of the International Mathematical Virtual Institute, vol. 8, no. 3, pp. 461-467, 2018.

[7] M. Munir and A. Shafiq, "A generalization of bi ideals in semirings," Bulletin of the International Mathematical Virtual Institute, vol. 8, no. 1, pp. 123-133, 2018.

[8] M. Henriksen, "Ideals in semirings with commutative addition," Amer. Math. Soc. Notices, vol. 6, no. 1, p. 321, 1958.

[9] D. R. La Torre, "On h-ideals and k-ideals in hemirings," Universitatis Debreceniensis, vol. 12, no. 2, pp. 219-226, 1965.

[10] S. I. Baik and H. S. Kim, "On fuzzy k-ideals in semirigs," Korean Journal of Mathematics, vol. 8, no. 2, pp. 147-154, 2000.

[11] R. Anjum, S. Ullah, Y. M. Chu, M. Munir, N. Kausar, and S. Kadry, "Characterizations of ordered h-regular semirings by ordered h-ideals," AIMS Mathematics, vol. 5, no. 6, pp. 5768-5790, 2020.

[12] M. Shabir and R. Anjum, "Right k-weakly regular hemirings," Quasigroups and Related Systems, vol. 20, no. 1, pp. 97-112, 2012.

[13] M. Shabir and R. Anjum, "Characterizations of hemirings by the properties of their k-ideals," Applied Mathematics, vol. 4, no. 5, pp. 753-768, 2013.

[14] M. Shabir and R. Anjum, "On k-bi-ideals in hemirings," New Mathematics and Natural Computation, vol. 8, no. 3, pp. 323-341, 2012.

[15] I. R. Goodman, "Fuzzy sets as equivalence classes of possibility random sets," Fuzzy Sets and Possibility Theory: Recent Developments, 1982.
[16] R. Verma and B. D. Sharma, "Fuzzy generalized prioritized weighted average operator and its application to multiple attribute decision making," International Journal of Intelligent Systems, vol. 29, no. 1, pp. 26-49, 2014.

[17] R. Verma, "Cosine entropy and similarity measures for fuzzy sets," Konuralp Journal of Mathematics, vol. 3, no. 1, pp. 8393, 2015.

[18] R. Verma and S. Maheshwari, "A new measure of divergence with its application to multi-criteria decision making under fuzzy environment," Neural Computing and Applications, vol. 28, no. 8, pp. 2335-2350, 2017.

[19] R. Verma, "On generalized fuzzy divergence measure and their applications to multi-criteria decision making," Journal of Combinatorics \& System Sciences, p. 39, 2014.

[20] M. S. Sezen, "Controlled fuzzy metric spaces and some related fixed point results," Numerical Methods for Partial Differential Equations, vol. 37, no. 1, pp. 583-593, 2021.

[21] N. Alamgir, Q. Kiran, H. Aydi, and Y. U. Gaba, "On multivalued fuzzy contractions in extended b-metric spaces," Journal of Mathematics, vol. 2021, Article ID 5579991, 11 pages, 2021.

[22] K. Javed, F. Uddin, H. Aydi, M. Arshad, U. Ishtiaq, and H. Alsamir, "On fuzzy b-metric-like spaces," Journal of Function Spaces, vol. 2021, Article ID 6615976, 9 pages, 2021.

[23] K. Javed, F. Uddin, H. Aydi, A. Mukheimer, and M. Arshad, "Ordered-theoretic fixed point results in fuzzy b-metric spaces with an application," Journal of Mathematics, vol. 2021, Article ID 6663707, 7 pages, 2021.

[24] J. Zhou, W. Pedrycz, X. Yue, C. Gao, Z. Lai, and J. Wan, "Projected fuzzy C-means clustering with locality preservation," Pattern Recognition, vol. 113, p. 107748, 2021.

[25] D. Dubois and H. Prade, "Fuzzy sets and probability: misunderstandings, bridges and gaps," in [Proceedings 1993] Second IEEE International Conference on Fuzzy Systems, pp. 10591068, San Francisco, CA, USA, 1993.

[26] P. Z. Wang, Fuzzy Sets and Falling Shadows of Random Sets, Beijing Normal University, 1985.

[27] S. K. Tan, P. Z. Wang, and X. Z. Zhang, "Fuzzy inference relation based on the theory of falling shadows," Fuzzy Sets and Systems, vol. 53, no. 2, pp. 179-188, 1993.

[28] Y. B. Jun and M. S. Kang, "Fuzzy positive implicative ideals of BCK-algebras based on the theory of falling shadows," Computers \& Mathematics with Applications, vol. 61, no. 1, pp. 62-67, 2011.

[29] B. Yu and J. Zhan, "Characterizations of hemirings based on probability spaces," Journal of Applied Mathematics, vol. 2013, Article ID 716435, 9 pages, 2013.

[30] X. H. Yuan and E. S. Lee, "A fuzzy algebraic system based on the theory of falling shadows," Journal of Mathematical Analysis and Applications, vol. 208, no. 1, pp. 243-251, 1997. 